\title{
Stroke-related erectile dysfunction: Impact of the cerebrovascular stroke site on the degree of erectile dysfunction, total testosterone and prolactin levels
}

\author{
Original \\ Article \\ Emad Eldien Kamal', Ahmed A. Abdel-Mageed ${ }^{1}$, Doaa A. Mahdy ${ }^{1}$,Tarek A. Rageh ${ }^{2}$, Aya \\ Y. Badran ${ }^{1}$ \\ Departments of ${ }^{1}$ Dermatology, Venereology and Andrology, ${ }^{2}$ Neuropsychiatry, Faculty of \\ Medicine, Assiut University, Assiut, Egypt
}

\begin{abstract}
Background: Stroke is one of the major causes of permanent disability and mortality in the aging population worldwide. Reduced libido, and poor erection and ejaculation are among common poststroke sexual dysfunctions, which is due to multiple factors, including both organic and psychosocial factors.

Objective: We designed the study in order to detect the presence of erectile dysfunction (ED) among male patients with stroke using the International Index of Erectile Function (IIEF-5) and correlate the site of the lesion with the degree of ED. Also, we evaluated the serum total testosterone and prolactin levels among those patients and correlated their levels with the degree of ED using IIEF-5.

Patients and Methods: Eighty male patients with ischemic cerebrovascular stroke were included in the study. The patients were diagnosed and divided according to the brain site lesion of cerebrovascular stroke into seven groups. The stroke patients completed the IIEF-5. Serum testosterone and prolactin levels were obtained and compared.

Results: The mean age of the patients was $55.35 \pm 3.68$ years. ED was detected in 53 patients representing $66.3 \%$ with variable degrees of severity (22.5\% mild, $6.3 \%$ mild to moderate, $7.5 \%$ moderate, and $30.0 \%$ severe), while $27(33.8 \%)$ patients had no ED. The mean testosterone level was lower than normal in basal ganglion affection and multiple areas affection only, while the mean prolactin level was higher than normal in all groups.

Conclusion: ED is a common problem that may follow cerebrovascular stroke. We do recommend that counseling by a trained professional should be made part of stroke rehabilitation.
\end{abstract}

Key Words: Cerebrovascular stroke, erectile dysfunction, international index of erectile function-5, prolactin, testosterone.

Received: 17 February 2020, Accepted: 09 March 2020.

Corresponding Author: Aya Y. Badran, Ph.D, Department of Dermatology and Venereology, Faculty of Medicine, Assiut University Hospital, Assiut, Egypt, Tel.: +201013244819, E-mail: aya_badran@yahoo.com.

ISSN: 2090-6048, September 2019 Vol. 9, No. 3

\section{INTRODUCTION}

Stroke is thought to be one of the major causes of permanent disability (paralysis, aphasia,or dysphasia)and mortality in the aging populationworldwide ${ }^{[1]}$. It is the third leading cause of death (after heart disease and cancer) with an incidence of 5.5 million persons/year ${ }^{[2]}$.

In 1988, the WHO Multinational Monitoring of Trends and Determinants in Cardiovascular Disease (MONICA) Project defined stroke as a syndrome with symptoms of focal or global disturbance affecting brain function, forat least $24 \mathrm{~h}$, which may leadto death in less than $24 \mathrm{~h}$, with no apparent cause other than of vascular origin ${ }^{[3]}$.

Irreversible brain insult occurs as a result of hypoxic or ischemic damage to the brain, which was mediated through multiple factors such as apoptosis, inflammation, oxidative damage, neuronal death, and others ${ }^{[4]}$.
Reduced sex drive (libido), and poor erection and ejaculation are among commonpoststroke sexual dysfunctions, dueto multiple factors, including both organic (lesion localization, premorbid medical conditions, and medications) and psychosocial (fear, low self-esteem, anxiety, and depression) factors ${ }^{[5]}$.

Diabetes mellitus, arterial hypertension, cigarette smoking, and dyslipidemia, which are the main risk factors for erectile dysfunction (ED), are also considered to bethe main risk factors for cerebrovascular stroke, especially in developed countries ${ }^{[6,7]}$. They exert their damaging effect through promoting arteriosclerotic changes with its subsequent dysfunction on the endothelium of the erectile penile tissue, which in turn leads to impairment of the vasodilation within the erectile penile tissue and accounts for vasculogenic $\mathrm{ED}^{[8]}$.

We designed the study on patients with cerebrovascular 
stoke in order to detect the presence of ED and its degree and to correlate the location of brain injury to the degree of ED. We also analyzed the relationship between the levels of both testosterone and prolactin and both the degree of $\mathrm{ED}$ and the site of brain affection.

\section{PATIENTS AND METHODS}

The study was a prospective hospital-based cross-sectional study which was performed at the Department of Dermatology, Venerology and Andrology, in collaboration with the Department ofNeuropsychiatry, Assiut, Egypt, during the period from April 2017 to May 2018. We included all male patients withdifferent types of ischemic cerebrovascular stroke who had attended the NeuropsychiatryDepartment during that period. Eighty male patients were included in the study. The concept of the study was explained to all participants and a writteninformed consent was obtained from each patient.The study protocol was approved by the Assiut Medical School Ethics Review Board (Ethics Committee N:IRB 17100862).

We excluded patients older than 70 years, patients on phosphodiesterase type 5-inhibitors or any other drugs modifying erectile function, patients with systemic diseases (diabetes mellitus, chronic liver, and kidney diseases), patients with hypogonadism, and patients having communication problems (severe aphasia, dementia, ordysarthria) due to their inability to be interviewed.

After complete medical history and meticulous clinical examination, complete neurologicalexamination by an expert neurologist, the stroke location was determined by either cranial computed tomography or MRI. All patients were subdivided into seven subgroups (from $A$ to $G$ ) according to the affected brain site.

Each patient was asked to complete the Arabic version of The International Index of Erectile Function (IIEF-5) for assessment of male sexual function. The IIEF-5 addresses thefive domains of sexual functions (erectile function, orgasmic function, sexual desire, intercourse satisfaction, and overall satisfaction). The IIEF-5 score is the sum of the responses to the five items; thus, the score ranges between 5 and 25as follows: 5-7 (severe ED), 8-11 (moderate ED), 12-16 (mild to moderate ED), 17-21 (mild ED), and 22-25 (no ED).

Venous blood samples were obtained early morning from all patients to measure total testosterone levelsby enzyme-linked immunosorbent assay using Thermo Scientific Multiskan FC kits (Finland, Vantaa). Normal value ranges from 2.50 to $11.00 \mathrm{ng} / \mathrm{ml}$. Also, prolactin levelswere measured by enzyme-linked immunosorbent assay using Thermo Scientific Multiskan FC kits (Finland). Normal value ranges from 4.04 to $15.20 \mathrm{ng} / \mathrm{ml}$.

\section{Statistical analysis}

Analysis of the data was performed using the Statistical Program for the Social Sciences (SPSS.18.0, SPSS Inc., Chicago, Illinois, USA) software program. Quantitative data were expressed as mean $\pm \mathrm{SD}$. Qualitative data were expressed as frequency and percentage.Independentsamples ttest of significance was used when comparing between two means. Pearson's correlation coefficient (r) test was used for correlating data, and one-way analysis of variance when comparing between more than two means. A $P$ value of less than 0.05 was considered statistically significant. $P$ value less than 0.001 was considered as highly significant, while $P$ value more than 0.05 was considered insignificant.

\section{RESULTS}

Our study included 80 male patients with ischemic cerebrovascular stroke. The mean age of thestroke patients was $55.35 \pm 3.68$ years and the mean duration ofstroke in them was $12.72 \pm 4.85$ months. The patients were subdivided into seven subgroups according to the affected brain site (Table1) as follows:

Group A included 17) patients with middle cerebral artery (MCA) affection. The mean age of those patients is $55.71 \pm 3.37$ years and the mean duration of stroke is $18.24 \pm 7.27$ months. The mean testosterone is $4.45 \pm 4.43 \mathrm{ng} / \mathrm{ml}$ and the mean prolactin is $20.48 \pm 13.06 \mathrm{ng} / \mathrm{ml}$.

Group Bincluded 13 patients with posterior cerebral artery (PCA) affection. The mean age of those patients is $56.23 \pm 3.77$ years andthe mean duration of stroke is $13.59 \pm 5.88$ months. The mean testosterone is $4.26 \pm 3.73 \mathrm{ng} / \mathrm{ml}$ and the mean prolactin is $20.04 \pm 13.07 \mathrm{ng} / \mathrm{ml}$.

Group C included 12 patients withanterior cerebral artery (ACA) affection. The mean age of those patients is $55.58 \pm 3.58$ years and the mean duration ofstrokeis $11.06 \pm 5.71$ months. The mean testosterone is $4.23 \pm 3.67 \mathrm{ng} / \mathrm{ml}$ and the mean prolactin is $20.03 \pm 11.40 \mathrm{ng} / \mathrm{ml}$.

Group D included 11 patients with brain stem affection. The mean age of thosepatients is $54.00 \pm 2.72$ years andthe mean duration of stroke is $12.58 \pm 4.73$ months. The mean testosterone is $3.44 \pm 3.96 \mathrm{ng} / \mathrm{ml}$ and the mean prolactin is $21.17 \pm 13.19 \mathrm{ng} / \mathrm{ml}$.

Group E included 10 patients with cerebellum affection. The mean age of those patients is $52.90 \pm 2.47$ years and the mean duration ofstroke is $10.44 \pm 3.42$ months. The mean testosterone is $4.75 \pm 3.55 \mathrm{ng} / \mathrm{ml}$ and the mean prolactin is $16.82 \pm 12.10 \mathrm{ng} / \mathrm{ml}$. 
Group $\mathrm{F}$ included nine patients with basal ganglion affection. The mean age of those patients is $53.44 \pm 3.91$ years and the mean duration of stroke is $9.81 \pm 4.56$ months. The mean testosterone is $2.06 \pm 2.85 \mathrm{ng} / \mathrm{ml}$ and the mean prolactin is $28.37 \pm 13.20 \mathrm{ng} / \mathrm{ml}$.
Group $\mathrm{G}$ included eight patients with multiple areas affection. The mean age of those patients is $60.00 \pm 2.07$ years and the mean duration of stroke is $16.55 \pm 6.80$ months. The mean testosterone is $0.64 \pm 0.28 \mathrm{ng} / \mathrm{ml}$ and the mean prolactin is $44.79 \pm 2.01 \mathrm{ng} / \mathrm{ml}$.

Table 1: Descriptive data of all patients with cerebrovascular stroke.

\begin{tabular}{lcccc}
\hline Groups & Brain site & No. $(\mathrm{N}=80)[\mathrm{n}(\%)]$ & Age (years) & Duration (months) \\
\hline Group A & MCA & $17(21.3)$ & $55.71 \pm 3.37$ & $18.24 \pm 7.27$ \\
Group B & PCA & $13(16.3)$ & $56.23 \pm 3.77$ & $13.59 \pm 5.88$ \\
Group C & ACA & $12(15.0)$ & $55.58 \pm 3.58$ & $11.06 \pm 5.71$ \\
Group D & Brain stem & $11(13.8)$ & $54.00 \pm 2.72$ & $12.58 \pm 4.73$ \\
Group E & Cerebellum & $10(12.5)$ & $52.90 \pm 2.47$ & $10.44 \pm 3.42$ \\
Group F & Basal ganglion & $9(11.3)$ & $53.44 \pm 3.91$ & $9.81 \pm 4.56$ \\
Group G & Multiple lesions & $8(10.0)$ & $60.00 \pm 2.07$ & $16.55 \pm 6.80$ \\
\hline
\end{tabular}

ACA, anterior cerebral artery; MCA, middle cerebral artery; PCA, posterior cerebral artery.

The scores of the IIEF-5questionnaire showed that $53(66.3 \%)$ patients had ED with varying severity $(22.5 \%$ mild, $6.3 \%$ mild to moderate, $7.5 \%$ moderate, and $30.0 \%$ severe), while no evidence of ED was detected in 27 $(33.8 \%)$ patients. The scores of the IIEF-5 questionnaire in all groups is presented in Table 2, Fig.1.

A comparison between the IIEF-5 in all stroke patients was performed; and revealed a statistically significant difference between the IIEF-5 score in each group (Table 3).

Regarding endocrinal evaluation of all patients, measurement of total testosterone level showed that the mean testosterone level in stroke groups was $3.64 \pm 3.72 \mathrm{ng} / \mathrm{ml}$ (Table 4), while that of serum prolactin levels was $23.30 \pm 14.00 \mathrm{ng} / \mathrm{ml}$ (Table 5).

Correlation analysis of the IIEF-5 score with the age of patients, serum testosterone, and prolactin levels showed thatthere was statistically significant positive correlation between IIEF-5 score and serum testosterone, while significant negative correlation between IIEF-5 score and bothage and serum prolactin (Table 6).

Another correlation analysis was performed between age with serum testosterone and prolactin levels, and showed that there was statistically significant negative correlation is present between age and serum testosterone (r $=-0.414, P=0.000$ ), while statistically significant positive correlation between age and serum prolactin $(\mathrm{r}=0.511, P=0.000)$.

Table 2: The International Index of Erectile Function-5 scores in allpatient groups

\begin{tabular}{lcccccccc}
\hline & $\begin{array}{c}\text { Group A } \\
(\mathrm{N}=17) \\
{[\mathrm{n}(\%)]}\end{array}$ & $\begin{array}{c}\text { Group B } \\
(\mathrm{N}=13) \\
{[\mathrm{n}(\%)]}\end{array}$ & $\begin{array}{c}\text { Group C } \\
(\mathrm{N}=12) \\
{[\mathrm{n}(\%)]}\end{array}$ & $\begin{array}{c}\text { Group D } \\
(\mathrm{N}=11) \\
{[\mathrm{n}(\%)]}\end{array}$ & $\begin{array}{c}\text { Group E } \\
(\mathrm{N}=10) \\
{[\mathrm{n}(\%)]}\end{array}$ & $\begin{array}{c}\text { Group F } \\
(\mathrm{N}=9)[\mathrm{n} \\
(\%)]\end{array}$ & $\begin{array}{c}\text { Group G } \\
(\mathrm{N}=8)[\mathrm{n} \\
(\%)]\end{array}$ & $\begin{array}{c}\text { Total } \\
(\mathrm{N}=80) \\
{[\mathrm{n}(\%)]}\end{array}$ \\
\hline No ED & $7(41.2)$ & $5(38.5)$ & $5(41.7)$ & $4(36.4)$ & $5(50.0)$ & $1(11.1)$ & 0 & $27(33.8)$ \\
Mild & $3(17.6)$ & $4(30.8)$ & $3(25.0)$ & $2(18.2)$ & $3(30.0)$ & $3(33.3)$ & 0 & $18(22.5)$ \\
Mild to moderate & 0 & $1(7.7)$ & $1(8.3)$ & $1(9.1)$ & $1(10.0)$ & $1(11.1)$ & 0 & $5(6.3)$ \\
Moderate & $1(5.9)$ & $2(15.4)$ & $1(8.3)$ & $2(18.2)$ & 0 & 0 & 0 & $6(7.5)$ \\
Severe & $6(35.3)$ & $1(7.7)$ & $2(16.7)$ & $2(18.2)$ & $1(10.0)$ & $4(44.4)$ & $8(100.0)$ & $24(30.0)$ \\
\hline
\end{tabular}

IIEF-5, The International Index of Erectile Function. 


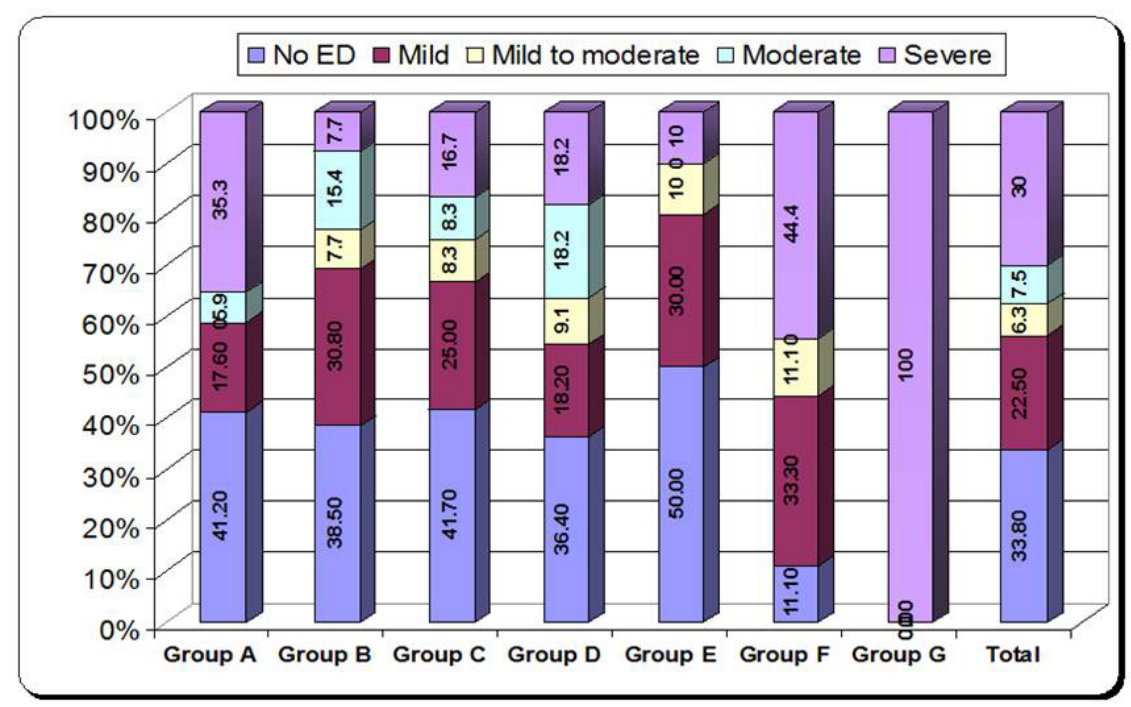

Fig. 1: Distribution of ED percentage inpatients with stroke.ED, erectile dysfunction.

Table 3: Comparison between the mean, median, and rangeof International Index of Erectile Function-5 in all patient groups.

\begin{tabular}{|c|c|c|c|c|c|c|c|c|}
\hline \multirow{2}{*}{ IIEF-5 } & \multicolumn{7}{|c|}{ Brain Site } & \multirow{2}{*}{$P$ value } \\
\hline & Group A & Group B & Group C & Group D & Group E & Group F & Group G & \\
\hline Mean \pm SD & $15.5 \pm 8.2$ & $17.2 \pm 6.4$ & $16.9 \pm 6.5$ & $15.7 \pm 7.2$ & $18.6 \pm 5.6$ & $13.0 \pm 7.2$ & $6.4 \pm 0.7$ & 0.037 \\
\hline Median (range) & $19(5-25)$ & $18(5-24)$ & $19.5(6-23)$ & $17(5-24)$ & $20(5-24)$ & $13(5-23)$ & $6.5(5-7)$ & \\
\hline
\end{tabular}

Comparison between the IIEF-5 in all patient groups.

IIEF-5, International Index of Erectile Function.

Statistically significant difference $(P<0.05)$ Kruskal-Wallis test

Table 4: Serum total testosterone level in all patient groups

\begin{tabular}{lc}
\hline Groups & Testosterone $(\mathrm{ng} / \mathrm{ml})$ \\
\hline Total patients & $3.64 \pm 3.72$ \\
Group A & $4.45 \pm 4.43$ \\
Group B & $4.26 \pm 3.73$ \\
Group C & $4.23 \pm 3.67$ \\
Group D & $3.44 \pm 3.96$ \\
Group E & $4.75 \pm 3.55$ \\
Group F & $2.06 \pm 2.85$ \\
Group G & $0.64 \pm 0.28$ \\
$P$ value & 0.030 \\
\hline
\end{tabular}

Data are expressed in the form of mean \pm SD.
Table 5: Serum prolactin level inall patient groups

\begin{tabular}{lc}
\hline Goups & Prolactin $(\mathrm{ng} / \mathrm{ml})$ \\
\hline Total patients & $23.30 \pm 14.00$ \\
Group A & $20.48 \pm 13.06$ \\
Group B & $20.04 \pm 13.07$ \\
Group C & $20.03 \pm 11.40$ \\
Group D & $21.17 \pm 13.19$ \\
Group E & $16.82 \pm 12.10$ \\
Group F & $28.37 \pm 13.20$ \\
Group G & $44.79 \pm 2.01$ \\
$P$ value & 0.001 \\
\hline
\end{tabular}

Data are expressed in the form of mean \pm SD. 
Table 6: Correlation of The International Index of Erectile Function-5) with age, testosterone level, and prolactin levels.

\begin{tabular}{lcc}
\hline \multirow{2}{*}{ Variables } & IIEF-5 & r value \\
\cline { 2 - 3 } Age & value & 0.001 \\
Testosterone level & -0.474 & 0.003 \\
Prolactin level & 0.962 & 0.002 \\
\hline
\end{tabular}

IIEF-5, International Index of Erectile Function.

\section{DISCUSSION}

Various types of disabilities are known to occur following cerebrovascular stroke, which include physical problemssuch as walking difficulties, aphasia, etc.and psychological problems such as depression, low selfesteem., etc., which in turn negatively affect the overall daily activities, including sexual activity ${ }^{[9,10]}$. A plenty of studies and reviewshave proven that sexual function is disturbed in poststroke patients ${ }^{[9,11-14]}$; however, few studies have assessed and correlated hormonal profile in those patients.

During the last century, Monga et al..$^{[15]}$, and Korpelainen et al. ${ }^{[16]}$ reported ED in 62 and $75 \%$ of post-stroke patients, respectively. Then, during the current century, Kimura et $a l .{ }^{[9]}$ reported ED in $58.6 \%$ of male stroke patients, followed byBener et al. ${ }^{[11]}$ who reported ED in $48.3 \%$ of male stroke patients, lastly, Koehn et al. ${ }^{[7]}$ reported ED in $78.9 \%$ of stroke patients.

In our study, we included 80 patients with stroke patients, the mean age of them was $55.35 \pm 3.68$ years. A similar study by Purwata et al. ${ }^{[17]}$, who included 74 stroke patients with stroke. They found that the mean age of poststroke patients sampled was $52.19 \pm 4.37$ years. Also, the study conducted byAkinpelu et al. ${ }^{[18]}$ found that the mean age of poststroke patients sampled was 59.4 \pm 9.49 years.

In our study, we found that among 80 patients with stroke, $53(66.3 \%)$ patients suffered from ED with different grades of severity. According to the result of IIEF-5, the grades of severity were as follows: $24(30.0 \%)$ patients had severe degree of ED, six $(7.5 \%)$ patients had moderate degree of ED, five $(6.3 \%)$ patients had mild-to-moderate degree of ED, and 18(22.5\%) patients had mild degree of ED. These results were in accordance to the results of Koehn et al. ${ }^{[7]}$ who found that $20(35.1 \%)$ patients had severe degree of ED; four (7\%) patients had moderate ED; four $(7 \%)$ patientshad mild-to-moderate degree of ED; and $17(29.8 \%)$ patients had mild degree of ED.

Studies explained the occurrence of poststroke ED on the basis of disruption of central autonomic nerves and tracts ${ }^{[10]}$; physical impairmentsuch as motor weakness, spasticity, bladder, or bowel dysfunction ${ }^{[19]}$; psychological factors such as depression ${ }^{[16]}$, and lack ofself-confidence and self-esteem ${ }^{[20]}$.

In our study, we divided all patients into seven groups (A-G) according to the site of the brain injury;we found that affection of the MCA is the most common type of injury, followed by PCA, ACA, brain stem, cerebellum, basal ganglia, and lastly multiple areas affection was the least common type. Koehn et al. ${ }^{[7]}$ also found that MCA affection is the most common type of brain injury, followed by PCA, ACA, brain stem, cerebellar, basal ganglia, and lastly multiple areas affection.

Regarding the percentage of ED in all seven groups, it was as follows: $58.8,61.5,58.3,63.7,50,88.9$, and $100 \%$ for group A, B, C, D, E, F, and G, respectively, in our study, while in the study performed by Koehn et al. ${ }^{[7]}$ it was as follows: 78.8, 80.0, 100, 100, 80, 40, and 100 for patients with MCA, PCA, ACA, basal ganglia infarction, brain stem infarction, cerebellar and multiple area affection, respectively.

The medial and the inferior frontal lobes, which are both supplied by the MCA, are among the cortical areas contributing to erection ${ }^{[21]}$, so ischemia affecting these MCA-supplied autonomic structures will affect the erectile function, thus explaining the high prevalence of ED in our patients with MCA stroke.

As normal erection depends on contribution from ACA-supplied centers (as the anterior cingulate gyrus, the nucleusaccumbens, the fornix, and the medial preoptic area and paraventricular nuclei of the hypothalamus), we found that $58.3 \%$ of patients with ACA affection develop $\mathrm{ED}^{[21]}$.

Regarding patients with PCA affection, ED was detected in $61.5 \%$ of them. This occurs due to deterioration of the functions of the thalamic nuclei, the midbrain ventral tegmental area, substantia nigra, and afferent and efferent structures, which mediate arousal and erection ${ }^{[21]}$.

In our study and in patients with brain stem affection, ED was present in $63.7 \%$ of patients. This occurs due to interruption of signals that travel from the genital areas 
through thebrain stem toward supraspinal areas that control male sexual behavior.

Regarding patients with multiple area affection,our study results confirmed the presence of ED, which was detectedin all patients, and this was in accordance with the results of Jea-Hun et al. ${ }^{[22]}$ who claimed that patients with multiple brain lesions had a significant decrease in EDcomparedwith patients with one lesion.

Regarding the IIEF-5 score, we found a significant negative correlation between the IIEF-5 score andthe age of patients, which means that ED prevalence increases withincreasing patient age. This is in agreement with Koehn et $a l .{ }^{[7]}$ who established a negative correlation between age and IIEF-5 score in stroke patients.

The mean level of total testosterone blood samples taken from stroke patients was lower than normal ingroup $F$ and group $G$ basal ganglion affection and multiple areas affection only, while the mean prolactin level was higher than normal in all groups. This was in contrast with Reshmi et $a l .{ }^{[23]}$ who found that there was no association between endogenous testosterone and incident clinical stroke or ischemic brain changes in men.

The mean level of serum prolactinblood samples taken from the stroke patients was higherthan normal ranges in all groups. This is in agreement with Fikri et al. ${ }^{[24]}$ and Sankalp et al. ${ }^{[25]}$, who found 'thatmen with stroke had higherserum prolactinlevels than age-matched men without stroke.'

Raaz et al. ${ }^{[26]}$ suggested that prolactin contributes to the pathophysiology of ischemic cerebrovascular event through increasing activation of platelets.

\section{CONCLUSION}

We conclude that ED is a common complication that may follow cerebrovascular stroke, especially in patients with multiple areas affection. The presence of ED increases with increasing age, and multiplicity of brain lesions in poststroke patients and high prolactin level.Serum testosterone level decreases with the advancement of age and multiplicity ofbrain lesions.

We do recommend that counseling by an expert physician in that field should be a part of rehabilitation of poststroke patients.

\section{CONFLICT OF INTEREST}

Thre are no conlicts of interest.

\section{REFERENCES}

1. RosamondW, FlegalK, FurieK, GoA, GreenlundK, HaaseN, et al. Heart disease and stroke statistics - 2008 update: a report from the American
Heart Association Statistics Committee and Stroke 2008 Statistics Circulation 2008; 29;117(4):e25-146.

2. MukherjeeD, PatilCG. Epidemiology and the global burden of stroke. World Neurosurg 2011; 76:S85-S90.

3. TruelsenT, MähönenM, TolonenH, AsplundK, BonitaR, VanuzzoD. Trends in stroke and coronary heart disease in the WHO MONICA Project. Stroke2003; 34:1346-1352.

4. HuangJ, LiY, TangY, TangG, YangGY, WangY. CXCR4 antagonist AMD3100 protects bloodbrain barrier integrity and reduces inflammatory response after focal ischemia in mice. Stroke 2013; 44:190-197.

5. Calabrò RS, Gervasi G, Bramanti P. Male sexual disorders following stroke: an overview. Int J Neurosci2011; 121:598-604.

6. InmanBA, SauverJL, JacobsonDJ, McGreeME, NehraA, LieberMM, et al. A population-based, longitudinal study of erectile dysfunction and future coronary artery disease. Mayo Clin Proc2009; 84:108-113.

7. KoehnJ, CrodelC, DeutschM, KolominskyRabasPL, HoslKM, KorhrmannM, et al. Erectile dysfunction (ED) after ischemic stroke: association between prevalence and site of lesion. Clin Auton Res2015; 25:357-365.

8. ShamloulR, GhanemH. Erectile dysfunction. Lancet 2013; 381:153-165.

9. KimuraM, MurataY, ShimodaK, RobinsonRG. Sexual dysfunction following stroke. Compr Psychiatry2001; 42:217-222.

10. PistoiaF, GovoniS, BoselliC. Sex after stroke: a CNS only dysfunction?Pharmacol Res2006; 54:11-18.

11. BenerA, Al-HamaqAO, KamranS, Al-AnsariA. Prevalence of erectile dysfunction in male stroke patients, and associated co-morbidities and risk factors. Int Urol Nephrol2008; 40:701-708.

12. JungJH, KamSC, ChoiSM, JaeSU, LeeSH, HyunJS. Sexual dysfunction in male stroke patients:correlation between brain lesions and sexual function. Urology2008; 71:99-103.

13. DuitsA, Van OirschotN, Van OostenbruggeRJ, Van LankveldJ. The relevance of sexual responsiveness to sexual function in male stroke patients. JSex Med2009; 6:3320-3326.

14. ThompsonSB, WalkerL. Sexual dysfunction after stroke. underestimating the importance of psychological and physical issues. Webmed Centr Phys Med2011; 2:12. 
15. MongaTN, LawsonJS, InglisJ. Sexual dysfunction in stroke patients. Arch Phys Med Rehabil $1986 ; 67: 19-22$.

16. KorpelainenJ, NieminenP, MyllylaV. Sexual functioning among stroke patients and their spouses. Stroke1999; 30:715-719.

17. PurwataT, AndakaD, NuarthaA, WiratniC, SumadaK. Positive correlation between left hemisphere lesion and erectile dysfunction in post-stroke patients. Open Access Maced J Med Sci2019; 7:363-368.

18. AkinpeluAO, OsoseAA, OdoleAC, OdunaiyaNA. Sexual dysfunction in Nigerian stroke survivors, Afr Health Sci. 2013; 13(3):639-45.

19. McMahonCG. Erectile dysfunction.Intern Med J2014; 44:18-26.

20. ReesPM, FowlerCJ, MaasCP. Sexual function in menand women with neurological disorders. Lancet2007; 369:512-525.

21. TemelY, HafiziS, BeulsE, Visser-VandewalleV. The supraspinal network in the control of erection.
Expert Opin TherTargets2005; 9:941-954.

22. Jea-HunJ, Sung-ChulK, Sae-MinC, Sung-UkJ, Seung-HyunL, Jae-SeogHS. Sexual dysfunction in male stroke patients: correlation between brain lesions and sexual function. Urology 2007; 71:99-103.

23. ReshmiS, Gottesman RF, SheritaHG., KathrynA, AdrianD. Association Between endogenous testosterone and cerebrovascular disease in the ARIC Study (Atherosclerosis Risk in Communities). Stroke2016; 47:2682-2688.

24. FikriA, Ayşegül-AkagündüzE, Şule-Bilen๘. Assessment of prolactin associated platelet activation in ischemic stroke. J Clin Exp Investig2012; 3:1-6.

25. SankalpK, PallaviK, Muddeshwar MG. Serum prolactin level in patients of ischemic stroke. Int J Contemp Med Res2016; 3:77-83.

26. RaazD, WallaschofskiH, StumpfC. Increased prolactin in acute coronary syndromes as putative Coactivator of ADP-stimulated P-selectin expression. Horm Metab Res2006; 38:767-772. 